\title{
Adult hepatic fibropolycystic disease presenting as obstructive jaundice
}

\author{
Departments of \\ Gastroenterology and \\ Radiology, Western \\ General Hospital, \\ Edinburgh \\ A J K Williams \\ S R Wild \\ K R Palmer \\ Correspondence to: \\ Dr A J K Williams, \\ Department of \\ Gastroenterology, Western \\ General Hospital, Crewe \\ Road, Edinburgh EH4 2XU. \\ Accepted for publication \\ 20 November 1989
}

\author{
A J K Williams, S R Wild, K R Palmer
}

jaundice, severe pruritus, dark urine, and pale stools. He was on no medication and had no previous episodes of jaundice. Physical examination apart from jaundice and modest hepatomegaly was unremarkable.

An abdominal ultrasound scan showed extraand intrahepatic biliary dilatation but no cause was identified. A laparotomy was therefore performed. This showed a non-dilated common bile duct, an empty gall bladder, and ductal dilatation at the level of the porta hepatis, but no obstructive lesion was defined. An operative liver biopsy specimen showed severe cholestasis with bile duct multiplication and widening in the portal zones and focal hepatic necrosis consistent with extrahepatic biliary obstruction.

After this the patient was transferred for further help in his management. We noted he was well, although still jaundiced (bilirubin $32 \mu \mathrm{mol} / \mathrm{l}$ (normal range 3-14), alkaline phosphatase $685 \mathrm{U} / \mathrm{l}$ (normal range 30-140), aspartate aminotransferase $164 \mathrm{U} / 1$ (normal range 9-52)). His past medical history was unremarkable but his grandfather had died from uraemia caused by polycystic renal disease.

An endoscopic retrograde cholangiopancreatogram (ERCP) was performed. The pancreatogram was normal but the cholangiogram showed a smooth stricture $2 \mathrm{~cm}$ above the insertion of the cystic duct (Fig 1). The gall bladder was filled but no contrast medium passed through the stricture. An abdominal ultrasound scan showed a dilated biliary tree and a $4 \mathrm{~cm}$ diameter hepatic cyst adjacent to the porta hepatis. Some $30 \mathrm{ml}$ of clear fluid was aspirated under ultrasound guidance. Cytology of the aspirate showed ciliated columnar cells. No other cysts in any other organ (kidneys, spleen, pancreas) were identified.

The patient's jaundice resolved and eight weeks later a repeat ERCP showed a normal cholangiogram (Fig 2), and an abdominal ultrasound scan showed no biliary dilatation and a small $1.5 \mathrm{~cm}$ diameter cyst adjacent to the porta hepatis.

Twelve months later the patient is well, with normal liver function tests and no ultrasound evidence of an increase in the size of the hepatic cyst.

\section{Discussion}

Jaundice caused by duct obstruction is a rare complication of solitary and polycystic liver disease,${ }^{6}$ and has not previously been shown to resolve with percutaneous cyst aspiration.

Hepatic cystic disease is associated with cysts in other organs (kidney, pancreas, spleen), but this was not so in our patient.

Some $50 \%$ of patients have polycystic kid- 


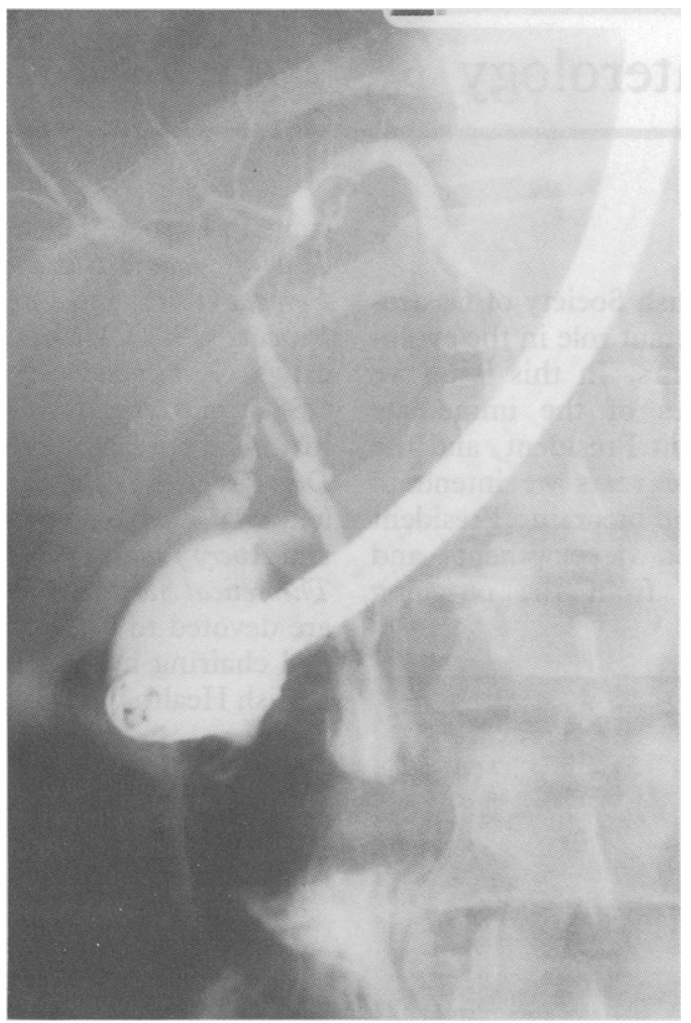

Figure 2: Repeat ERCP showed a normal cholangiogram.

neys. ${ }^{127}$ The grandfather of our patient had polycystic renal disease, but our patient had normal renal function and no cysts in the kidneys.

Although only one hepatic cyst was found in our patient the number of cysts present within the liver can vary from one to many in fibropolycystic disease, and in view of the family history of polycystic renal disease we regarded this patient as being within the spectrum of this condition. Solitary non-parasitic hepatic cysts are increas- ingly regarded as a variant of polycystic disease. ${ }^{\circledR}$

The hepatic cysts arise from defective embryological development of intrahepatic bile ducts and are lined by a secretory epithelium of cuboidal or columnar cells. Ciliated columnar cells were identified from the cystic fluid in our patient. Analysis of the cystic fluid indicates that it resembles that fraction of human bile which is secreted independently of the presence of bile salts. ${ }^{9}$

The cysts, although congenital, are formed by functioning secretory bile duct epithelium and gradually increase in size throughout childhood and early adult life to cause symptoms in the fourth or fifth decade. In this case the cyst reached a critical size which resulted in mechanical compression of the common hepatic duct and this was relieved by aspiration.

The cysts, if symptomatic, are best managed by percutaneous aspiration under ultrasound control, although they have a tendency to recur, and introduction of a sclerosant may delay this. ${ }^{10}$ The prognosis of the condition is governed by the presence of renal involvement.

1 Melrick PJ. Polycystic liver. Arch Pathol 1955; 59: 162-72.

2 Brown RAP. Polycystic disease of the kidneys and intracranial aneurysms. Glasgow Med F 1951; 32: 333-53.

Comfort MW, Gray HK, Dahlin DC, Whitesell FB. PolyComfort MW, Gray HK, Dahlin DC, Whitesell FB. Poly-
cystic disease of the liver - a study of 24 cases. Gastrocystic disease of the liver
enterology 1952; 20: 60-78.

4 Del Guercio E, Greco J, Kim KE, Chnitz J, Swartz C. Oesophageal varices in adult patients with polycystic kidney and liver disease. $N$ Engl F Med 1973; 289: 678-9.

5 Longmire WP, Mandiola SA, Gordon HE. Congenital cystic disease of the liver and biliary system. Ann Surg 1971; 174: 711-24.

6 Wittig JH, Burns R, Longmire WP. Jaundice associated with polycystic liver disease. Am $\mathcal{F}$ Surg 1971; 136: 383-6.

7 Dalgaard OZ. Bilateral polycystic disease of the kidneys. A follow-up of 284 patients with their families. Acta Med Scand 1957; suppl 328: 13-255.

8 Sherlock S. Diseases of the liver and biliary system. 7th edition. Oxford: Blackwell Scientific Publications, 1986: 435.

9 Patterson M, Gonzalez-Witale JC, Fagan CJ. Polycystic liver disease: a study of cyst fluid constituents Hepatology 1982; 2: $475-8$.

10 Saini S, Mueller PR, Ferrucci JT, Simeone JF, Wittenberg J, Butch RJ. Percutaneous aspiration of hepatic cysts does not provide definitive therapy. $A \mathcal{F} R$ 1983; 141: 559-60. 